\title{
Efficiency of Manual Scanning in Recovering Rare Cellular Events Identified by Fluorescence In Situ Hybridization: Simulation of the Detection of Fetal Cells in Maternal Blood
}

\author{
Ahmed Emad, ${ }^{1}$ Seemi Ayub, ${ }^{1}$ Oumar Samassékou, ${ }^{1}$ Marie-Chantal Grégoire, ${ }^{1}$ \\ Macoura Gadji, ${ }^{1}$ Aimé Ntwari, ${ }^{1}$ Josée Lamoureux, ${ }^{1}$ Francis Hemmings, ${ }^{1}$ Triantafyllos Tafas, ${ }^{2}$ \\ Michael W. Kilpatrick, ${ }^{2}$ Kada Krabchi, ${ }^{1}$ and Régen Drouin ${ }^{1}$ \\ ${ }^{1}$ Division of Genetics, Department of Pediatrics, Faculty of Medicine and Health Sciences, Universite de Sherbrooke, \\ QC, Canada J1H $5 \mathrm{~N} 4$ \\ ${ }^{2}$ Ikonisys Inc., 5 Science Park, Suite 1000, New Haven, CT, 06511, USA
}

Correspondence should be addressed to Régen Drouin, regen.drouin@usherbrooke.ca

Received 15 October 2011; Accepted 19 December 2011

Academic Editor: Brynn Levy

Copyright ( $) 2012$ Ahmed Emad et al. This is an open access article distributed under the Creative Commons Attribution License, which permits unrestricted use, distribution, and reproduction in any medium, provided the original work is properly cited.

\begin{abstract}
Fluorescence in situ hybridization (FISH) and manual scanning is a widely used strategy for retrieving rare cellular events such as fetal cells in maternal blood. In order to determine the efficiency of these techniques in detection of rare cells, slides of XX cells with predefined numbers (1-10) of XY cells were prepared. Following FISH hybridization, the slides were scanned blindly for the presence of XY cells by different observers. The average detection efficiency was $84 \%$ (125/148). Evaluation of probe hybridization in the missed events showed that $9 \%(2 / 23)$ were not hybridized, $17 \%(4 / 23)$ were poorly hybridized, while the hybridization was adequate for the remaining $74 \%$ (17/23). In conclusion, manual scanning is a relatively efficient method to recover rare cellular events, but about $16 \%$ of the events are missed; therefore, the number of fetal cells per unit volume of maternal blood has probably been underestimated when using manual scanning.
\end{abstract}

\section{Introduction}

Detection of rare cellular events has enormous potential in both cancer [1-3] and prenatal diagnosis [4-7]. The presence of fetal cells in maternal circulation generates a great amount of interest as a source of genetic material for noninvasive and risk-free diagnosis of aneuploidies and single gene disorders [8]. Instead of cell-free fetal DNA (cffDNA) in maternal plasma, fetal cells in the maternal blood can be an alternative approach for the development of a noninvasive method for prenatal diagnosis that accurately detects chromosome anomalies for two major reasons: (1) to work with pure fetal DNA material, which will allow specific characterization of fetal genome and (2) to have the whole genome of the fetus and not just part of it. The number of fetal cells is extremely low in maternal blood [9, 10]; therefore, enrichment, accurate identification and optimal timing of recovery are essential for their reliable use in prenatal diagnosis [11-15].
Fetal cells could be identified by targeting specific genetic marker exclusive for the fetal cells by molecular cytogenetic techniques such as fluorescence in situ hybridization (FISH) and primed in situ labeling (PRINS). Manual scanning is a commonly used strategy for retrieving these rare fetal cells from maternal blood.

Recent studies have indicated that fetal cells can be detected directly from the maternal blood without prior enrichment, to avoid losing fragile fetal cells, using techniques such as FISH and PRINS. Low-frequency predictions were given by these studies that recognized fetal cells only by the presence of a Y chromosome signal in male pregnancy $[4,5,9,16,17]$. Although these studies yielded important information concerning the number of circulating fetal cells in maternal blood, the results are possibly skewed by the detection efficiency of these rare events by cumbersome and time-consuming manual scanning. Therefore, automation will be required for widespread clinical use of fetal cells in 
prenatal diagnosis. Precision of manual scanning is crucial to validate adequately any automatic scanning device. However, the accuracy and reliability of locating these rare cells by manual scanning has never been evaluated.

The purpose of this study was to develop a robust protocol to assess the detection efficiency of rare events such as fetal cells in the maternal blood. We developed a strategy to spread a known number of XY cells in predefined areas on the slide and to detect these XY cells amongst thousands of XX cells. This strategy allows for evaluation of the detection efficiency of the manual scanning by knowing the exact number of XY cells and their exact location on the slides. In addition, the efficiency of the FISH technique in recovering rare cells can be evaluated by verification of the missed events and evaluation of the hybridization signals after scanning. Furthermore, this strategy has various potential applications as it could be used in the validation of automatic scanning and comparisons between different detection techniques.

\section{Materials and Methods}

2.1. Sampling. Three milliliters $(3 \mathrm{~mL})$ of heparinized peripheral blood were obtained from both male and female donors and rendered anonymous. Donors were healthy, nonpregnant adults between 20 and 35 years of age. Immediately after sampling, whole blood samples were dispensed into $250 \mu \mathrm{L}$ aliquots, washed with Hank's balanced salt solution, and harvested by standard cytogenetic techniques. For both $\mathrm{XX}$ and XY cells, small aliquots of fixed cell suspensions were prepared and stored at $-20^{\circ} \mathrm{C}$ until needed.

2.2. Spreading and Counting. Spreading of $2 \mu \mathrm{L}$ of diluted fixed XY nucleus suspension at one, two, or three predefined spots onto cleaned slides was performed in a modified Thermotron environmental control unit (CDS-5, Thermotron, Amsterdam, Netherlands) at $25^{\circ} \mathrm{C}$ and $36 \%$ humidity. All slides were encoded and stained with 4\% Giemsa solution (Harleco; EMB, Gibbstown, NJ) containing 4\% of Sorensen's phosphate buffer [18]. Two different observers scored the number of XY cells on each slide, blindly. Slides with more than 11 cells or with no concordance of cell counts between observers were excluded. Each Giemsa-stained target cell was located, imaged using the 100x objective on an Olympus BX61 microscope and coordinates were registered. The selected slides were then subjected to secondary spreading with XX nuclei suspension of $1.5 \times 10^{5}$ nuclei on top of XY spreading areas. In addition, $100 \% \mathrm{XX}$ and XY cell slides were spread as controls. A total of $148 \mathrm{XY}$ cells were distributed on 60 slides. For statistical analysis, the slides were divided in two categories: 30 slides with a range of 2 to $11 \mathrm{XY}$ cells on each slide and 30 slides with either 0 or 1 XY cell per slide.

2.3. FISH Procedure. Slides were first aged overnight at $37^{\circ} \mathrm{C}$, then, immersed in $2 \mathrm{xSSC}$ at $37^{\circ} \mathrm{C}$ for 30 minutes. Slides were dehydrated through a series of ethanol baths $(70 \%$, $80 \%, 100 \%)$. Conventional dual-color FISH was performed, using probes specific for chromosomes $\mathrm{X}$ and $\mathrm{Y}$ (CEP X: spectrum orange alpha-satellite and CEP Y: spectrum green
satellite-III; Vysis/ABBOTT Diagnostics, Downers Grove, IL) diluted $1: 100$ and $1: 300$, respectively, in cDenHyb-1 (Insitus Biotechnologies, Albuquerque, NM). The slides and the probes were codenatured at $75^{\circ} \mathrm{C}$ for 5 minutes before being sealed with rubber cement and placed in a humid chamber for hybridization at $37^{\circ} \mathrm{C}$ for 16 hours. Coverslips were then carefully removed and the slides were washed with a solution of $0.4 \mathrm{xSSC} / 0.3 \% \mathrm{NP}-40$ at $72^{\circ} \mathrm{C}$ for 2 minutes. A second wash was performed in a solution of $2 \mathrm{xSSC} / 0.1 \% \mathrm{NP}-$ 40 at room temperature for 3 minutes. After a final wash with distilled water, the slides were mounted in DAPI II $(0.1 \mathrm{M}$ Tris $\mathrm{pH} 8.0,90 \%$ glycerol, $1 \mathrm{mg} / \mathrm{mL}$-phenylenediamine, 0.01\% 4,6-diamidino-2-phenylindole (DAPI)).

2.4. Microscopic Observation. Manual scoring was performed blindly on an Olympus BX-61 fluorescent microscope equipped with appropriate filter sets. Fluorescence nucleus pictures were taken using a CCD camera and ISIS-2 software (Metasystems, Altlussheim, Germany). All slides were analyzed using the appropriate single band pass filter. Primary search was performed for Y-signal using spectrum green band filter (FITC). Suspected events were subsequently verified for the presence of single $\mathrm{X}$ chromosome-specific signal on a DAPI-stained nucleus under the appropriate spectrum red (TRITC) and blue (DAPI) filters. The number of detected XY cells per slide, cellular location, and imaging were recorded, along with time required for the scoring of each slide. All manual microscopy was performed at $1000 \mathrm{X}$ magnification. We also scored the hybridization status of the Y signals in 5000 cellular events distributed in the 5 control slides of $100 \%$ XY cells. The slides were stored in dark at $-20^{\circ} \mathrm{C}$ after the scanning process to avoid bleaching of signals.

In all cases, cells were considered to be positive if the following criteria were met: nuclei had two different fluorescent signal colors representing both the $\mathrm{X}$ and $\mathrm{Y}$ chromosomes, an intact nuclear border as indicated by DAPI staining, and presence of fluorescent signals only through appropriate filters. Cells in direct contact with each other were excluded. The hybridization signals were usually bigger and brighter than the background signals such as debris, fluorescent materials, or air bubbles that are not blocked by the filter. Most of these background signals could be excluded by bleed through signals that is, signals which appears in all filters.

2.5. Rehybridization Procedure (Re-FISH). A reverse color FISH was done for all recovered cells to confirm the identity of the cells. Evaluation of its reliability in confirming positive events was also recorded. For the re-FISH procedure, coverslips were removed by dipping the slides in a prewarmed $2 \mathrm{xSSC}$ bath, at $37^{\circ} \mathrm{C}$ for 10 minutes. The existing FISH probes were then removed by denaturing the slides with $70 \%$ formamide $/ 2 \mathrm{xSSC}$ at $73^{\circ} \mathrm{C}$ for 2 minutes 30 seconds. Slides were then dehydrated in successive ice-cold ethanol baths $(70 \%, 80 \%, 100 \%)$ and air-dried. Finally, slides were processed through a second round of FISH procedure using the opposite fluorochrome labeling (X probe in green and $\mathrm{Y}$ probe in orange) to produce the reverse color FISH pattern. 


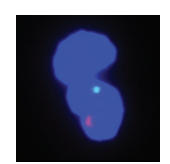

Cell detected by the observer

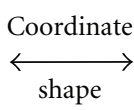

$$
\text { Previous XY cell }
$$
photo in Giemsa

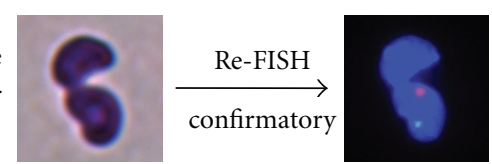

True positive cells evaluation of re-FISH

(a)
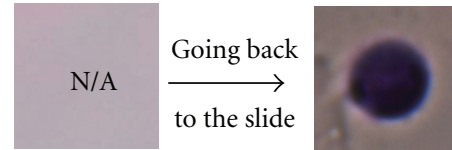

$$
\underset{\text { coordinate }}{\stackrel{\text { Shape }}{\longleftrightarrow}}
$$

$$
\text { Missed cell }
$$

by the observer

Previous XY cell

photo in Giemsa

False negative cells

evaluation of hybridization

(b)

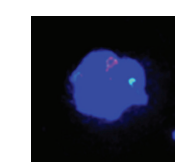

Extra cell detected by the observer

$$
\stackrel{\text { Shape }}{\stackrel{\text { coordinate }}{\longrightarrow}}
$$

Nonrecorded as $\mathrm{XY}$ cells in Giemsa

\section{Re-FISH}

$\overrightarrow{\text { informational }}$ for extra-cells

False positive cells evaluation of FP/TP

(c)

Figure 1: Schematic management of detected (a), missed (b), and extra cells (c). FP: false positive, TP: true positive, N/A: not acquired.

2.6. Analysis of Cellular Scanning. The slides were scanned blindly (without knowing the number of XY cells or their location on the slide) by one investigator, then the analysis was performed within 48 hours after scanning by another investigator. Following the scanning, the location (coordinates) and the shape of captured events were compared with that of the previously recorded Giemsa-stained photos. Depending on the results of the scanning, three different possibilities were observed (see Section 3).

2.7. Statistical Methods. The statistical analysis was performed using "proc reg" and "proc mixed" procedures of the Statistical Analysis System (SAS) software, version 9.1.3 (SAS Institute Inc., Cary, NC). Spearman's correlation was used to assess the process of the manual scanning and regression analysis curve was plotted to demonstrate the detection efficiency of the target events. Kruskal-Wallis test was done for the analysis of variance of status versus different observers. The odds ratio was calculated for determining the effect of hybridization on the detection efficiency. Index of Youden was used for assessment of the methodology.

\section{Results}

As our manual scanning approach was based on the finding of the Y signal first, we first tested the hybridization efficiency
TABLE 1: Interpretation of cellular events according to the concordance of the FISH images resulting from scanning and previously taken Giemsa images.

\begin{tabular}{lcc}
\hline Interpretation of cellular event & FISH photo & Giemsa photo \\
\hline $\begin{array}{l}\text { Retrieved XY target } \\
\text { (true positive cell) }\end{array}$ & Present & Present \\
Missed event & Absent & Present \\
Extra cell detected & Present & Absent \\
\hline
\end{tabular}

of the Y chromosome centromere probe. We found that the hybridization efficiency of the Y probe in 5,000 pure XY cells, processed by conventional FISH, was $99.1 \%(4,955 / 5,000)$. The hybridization was adequate in $97.3 \%(4,865 / 5,000)$ of cells, whereas the remaining $1.8 \%(90 / 5,000)$ showed poor hybridization signals.

Next, we evaluated the retrieval of rare cellular events by a manual scanning-based FISH method, using an approach, which is summarized in Figure 1 and Table 1. All slides were screened at 1000x for the Y-signal for primary detection, with subsequent confirmation of an X chromosome-specific signal on a DAPI-stained nucleus, using the appropriate band pass filters. Comparison of the location (coordinates) and the shape of captured events with that of the previously recorded Giemsa-stained photos resulted in three possible results (Figure 1 and Table 1). The first one is when a 
captured fluorescent image matched with a previously taken Giemsa photo, in which case the cell was scored as a recovered event (Figure 2). The second possibility is when no corresponding fluorescent image was found for a previously recorded Giemsa one, in which case the cell was scored as a missed event. In these circumstances, retrieval of the cell, using the coordinates and shape of the recorded Giemsa image, and evaluation of its hybridization efficiency were performed. Cells were scored as either a missed event or a hybridization failure. In the third scenario, a captured fluorescent image had no corresponding Giemsa one. ReFISH was then used to score the cell as either a true positive or false positive. Table 2 summarizes the results obtained by manual scanning. Two observers A and B blindly scanned 18 and 42 slides, respectively, in order to retrieve $148 \mathrm{XY}$ positive cells among around $90 \times 10^{5} \mathrm{XX}$ cells, distributed on 60 slides, with an average of $1.5 \times 10^{5} \mathrm{XX}$ cells per slide. The overall detection rate of the true positives was $84.5 \%$ (125 out of the $148 \mathrm{XY}$ cells). The remaining 23 missed cells were considered false negatives. Individually, observers $\mathrm{A}$ and $\mathrm{B}$ detected 25 out of the $35 \mathrm{XY}$ cells $(71.4 \%)$ and 100 out of the $113 \mathrm{XY}(88.5 \%)$, respectively (Figure $3(\mathrm{a})$ ). The variance analysis did not show a statistical difference between the two observers $(P=0.606)$. Therefore, the combined findings of these two observers were used to assess the sensitivity, specificity, and efficiency of rare events detected by the manual scanning and FISH technique. We found that the specificity and sensitivity for detection of XY cells were $99.9 \%$ and $84.5 \%$, respectively, with a positive predictive value of $97 \%$. In the same vein, by using the Spearman's correlation, we found high correlation between the detected cells and the number of predefined target cells per slide $(C . C=0.947$, $P<0.001)$. Regression analysis was plotted to demonstrate the relation of the detected cells versus the true number of XY cells (Figure 3(b)). Furthermore, a high index of Youden at 0.85 confirmed the efficiency of our approach. In summary, the manual scanning is a reliable method to detect rare events hybridized using the FISH technique.

To gain deeper insight into the causes of the occurrence of false-negative events, we evaluated missed cells after scanning. Of the 23 missed cells, 2 cells $(8.7 \%)$ were not hybridized for the $\mathrm{Y}$ chromosome, 4 cells $(17.4 \%)$ were poorly hybridized and the hybridization was adequate in the remaining 17 cells $(73.9 \%)$. Thus, manual scanning and the related FISH procedure were responsible for the occurrence of $73.9 \%$ and $26.1 \%$ of the false-negative events, respectively. The major cause of false-negative cells is the manual scanning and human fatigue. The long scanning time, which was estimated to be on average 150 minutes, might be one of the reasons for the occurrence of false negatives due to the manual scanning. The second cause of false negatives is defective hybridization, which could be attributed to insufficient hybridization at the target site or fading of the fluorescent signals (Figure 4). The latter is highlighted by the higher prolonged automatically adjusted exposure time for the $\mathrm{Y}$ probe channel ( $>0.72$ second) in contrast to an average of 0.32 seconds for the detected cells. Next, we investigated if the percentage of cells with inadequate hybridization could be due to a drawback of
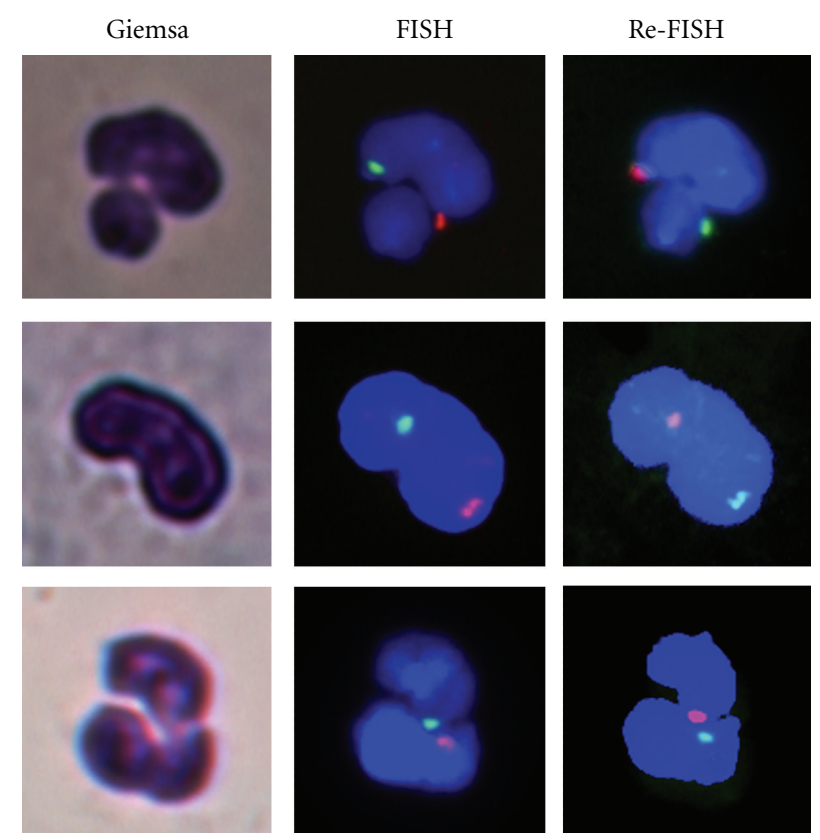

FIGURE 2: Example of Giemsa, FISH and re-FISH images of three detected events.

the double spreading and Giemsa staining procedure. We compared the hybridization efficiency of the $\mathrm{Y}$ probe on pure XY cells, without double spreading and Giemsa staining (see above), to that obtained from our FISH procedure on the prespread XY cells and we did not find any statistical significant difference of the percentage of nonhybridized $(P=0.3938)$ and poorly hybridized $(P=0.1797)$ cells of both groups. This led us to exclude the possibility of a procedure-related effect on the hybridization efficiency.

To evaluate extra cells and exclude false-positive events, we performed re-FISH on all detected cells, including the predefined XY cells and six additional cells. Evaluation of its reliability was measured on the detected predefined XY cells. Re-FISH gave an unambiguous reverse color pattern in $90.4 \%(113 / 125)$ of detected XY cells. Detected extra cells did not show the expected reversed signal pattern after reFISH hybridization except for three extra cells (two in slide AMP-22 and one in slide SMP-51) as shown in Table 2. Interestingly, these three cells were inside the predefined area of spreading, very close to other positive target cells in the corresponding slides. It appeared that they were missed in the original Giemsa-stained target counting step. These three events were included in the positive XY spread cells on the slides in the final tabulation for the sake of statistical analysis. Therefore, re-FISH is a highly reliable approach, which can help to exclude false-positive events.

\section{Discussion}

In this study, we develop a robust protocol to validate the detection of rare events by a manual scanning and FISH technique. We found that manual scanning and FISH allows the detection of 1-10 targeted cells, among a total of $1.5 \times$ 
TABLE 2: Results obtained by manual scanning.

\begin{tabular}{|c|c|c|c|}
\hline Slide & Spread XY cells & Detected cells & Missed cells \\
\hline AMP-9 & 1 & 1 & 0 \\
\hline AMP-10 & 4 & 4 & 0 \\
\hline AMP-11 & 2 & 0 & 2 \\
\hline AMP-12 & 5 & 4 & 1 \\
\hline AMP-13 & 5 & 2 & 3 \\
\hline AMP-14 & 2 & 2 & 0 \\
\hline AMP-18 & 3 & 3 & 0 \\
\hline AMP-19 & 0 & 0 & 0 \\
\hline AMP-20 & 1 & 1 & 0 \\
\hline AMP-21 & 1 & 1 & 0 \\
\hline AMP-22 & $3+2 E^{*}$ & 4 & 1 \\
\hline AMP-23 & 0 & 0 & 0 \\
\hline AMP-24 & 1 & 1 & 0 \\
\hline AMP-25 & 1 & 0 & 1 \\
\hline AMP-26 & 3 & 1 & 2 \\
\hline AMP-27 & 0 & 0 & 0 \\
\hline AMP-28 & 1 & 1 & 0 \\
\hline AMP-29 & 0 & 0 & 0 \\
\hline SMP-10 & 4 & 3 & 1 \\
\hline SMP-11 & 11 & 10 & 1 \\
\hline SMP-12 & 2 & 2 & 0 \\
\hline SMP-13 & 11 & 9 & 2 \\
\hline SMP-24 & 5 & 5 & 0 \\
\hline SMP-25 & 3 & 3 & 0 \\
\hline SMP-26 & 2 & 2 & 0 \\
\hline SMP-27 & 3 & 3 & 0 \\
\hline SMP-28 & 1 & 1 & 0 \\
\hline SMP-29 & 5 & 4 & 1 \\
\hline SMP-30 & 6 & 6 & 0 \\
\hline SMP-31 & 3 & 3 & 0 \\
\hline SMP-32 & 6 & 5 & 1 \\
\hline SMP-33 & 7 & 6 & 1 \\
\hline SMP-34 & 6 & 5 & 1 \\
\hline SMP-35 & 4 & 3 & 1 \\
\hline SMP-36 & 2 & 2 & 0 \\
\hline SMP-37 & 1 & 1 & 0 \\
\hline SMP-38 & 3 & 3 & 0 \\
\hline SMP-39 & 2 & 2 & 0 \\
\hline SMP-40 & 5 & 5 & 0 \\
\hline SMP-41 & 4 & 3 & 1 \\
\hline SMP-42 & 0 & 0 & 0 \\
\hline SMP-43 & 3 & 3 & 0 \\
\hline SMP-44 & 1 & 1 & 0 \\
\hline SMP-45 & 0 & 0 & 0 \\
\hline SMP-46 & 1 & 1 & 0 \\
\hline SMP-47 & 0 & 0 & 0 \\
\hline
\end{tabular}

TABLE 2: Continued.

\begin{tabular}{lccc}
\hline Slide & Spread XY cells & Detected cells & Missed cells \\
\hline SMP-48 & 2 & 2 & 0 \\
SMP-49 & 0 & 0 & 0 \\
SMP-50 & 1 & 1 & 0 \\
SMP-51 & $1+1 \mathrm{E}^{*}$ & 2 & 0 \\
SMP-52 & 0 & 0 & 0 \\
SMP-53 & 0 & 0 & 0 \\
SMP-54 & 0 & 0 & 0 \\
SMP-55 & 3 & 3 & 0 \\
SMP-56 & 0 & 0 & 0 \\
SMP-57 & 1 & 1 & 0 \\
SMP-58 & 0 & 0 & 0 \\
SMP-59 & 1 & 0 & 1 \\
SMP-60 & 1 & 0 & 1 \\
SMP-61 & 1 & 0 & 1 \\
\hline
\end{tabular}

$\mathrm{E}^{*}$, extra true positive cells confirmed by re-FISH.

$10^{5}$ cells, with $99.9 \%$ of specificity, $84.5 \%$ sensitivity, and a positive predictive value of $97 \%$. In addition, we found that this method is highly reliable and efficient with high Youden index of 0.85. Moreover, we determined the rate of falsenegative and false-positive events and the inherent causes of their occurrence.

The experimental design, which involved the assessment of slides containing known numbers of predefined rare target cells, allowed, for the first time, the retrieval and evaluation of hybridization of false-negative or missed events. Our results indicated that the manual scanning process is responsible for $73.9 \%$ of false-negative events while the remaining $26.1 \%$ was due to the FISH technique. The fatigue generated by the long time of scanning, on average 150 minutes per slide, and the screening of low numbers of small dots among thousands, might be some reasons of the occurrence of false negatives. These factors can be overcome by the development and validation of automatic scanning to search for these kinds of rare events. The second cause of false-negative events was the FISH technique, responsible for missing 4\% of the target cells and this percentage was not statistically different from the efficiency of the $\mathrm{Y}$ centromere probe. The diffuse and weak Y signal was mostly responsible for the FISH drawback. Diffused signals can be explained by chromatin extension forming chromatin fibers, which links two or more condensed domains of chromatin. These fibers usually show a very weak signal, which fades faster than a normal one [19]. The nature of defective signals resulting from overdecondensed chromatin points to the importance of prehybridization steps in the FISH technique. However, more effort should be oriented toward the reduction of false-negative events due to manual scanning, which can be overcome by the development of an automatic scanning system.

Interestingly, data collected in this study confirmed the reliability and accuracy of our previous methodology using manual scanning for the determination of the frequency 


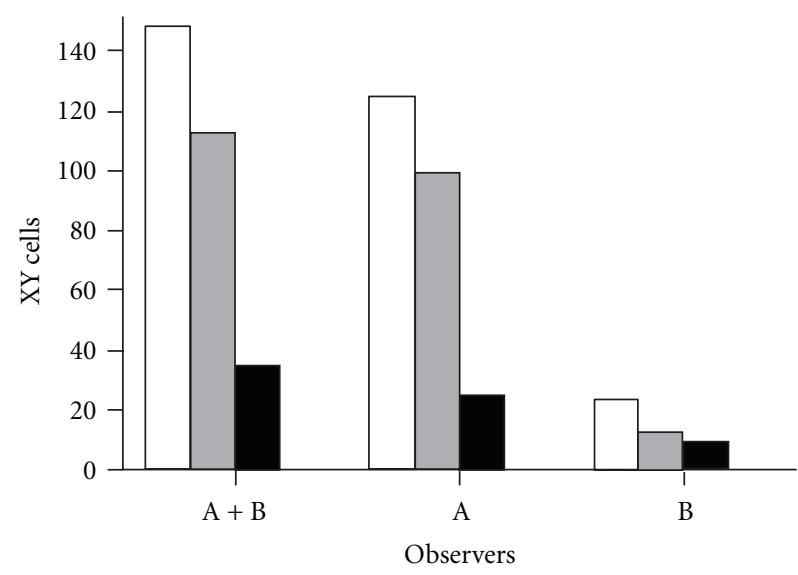

(a)



(b)

FIGURE 3: Comparison between detected cells and the true number of XY cells. (a) Summary of true (white bars), detected (grey bars) and missed (black bars) cells reported for observer A and observer B. (b) Regression analysis represents the correlation between these data.
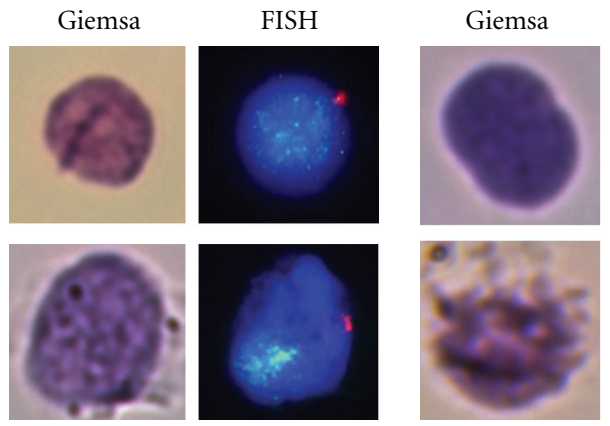

(a)
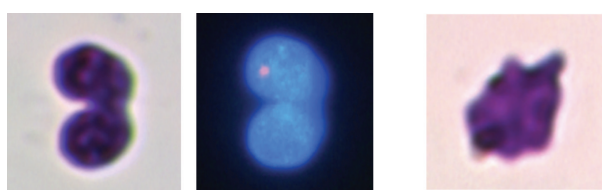

(b)

FIGURE 4: Giemsa and corresponding FISH photos of missed events due to inadequate hybridization (a) or nonhybridization events (b).

of fetal cells in maternal blood. Using this methodology, we previously located a median of 2 to 6 fetal cells per milliliter of maternal blood in the second trimester of normal pregnancy between 18 and 22 weeks [9]. This number is increased by 3 to 5 times in cases of Down's syndrome $[4,5]$. Similar increases were also detected in different types of aneuploidies $[4,5]$. According to our study, the number of detected fetal cells seemed to be underestimated by an average of $16 \%$ due to the occurrence of false-negative cells. Thus, these missing cells can be largely recovered by a robust automatic scanning, increasing the likelihood of the development of noninvasive prenatal diagnosis in future.

Our findings lay the groundwork for the validation of automatic scanning for the detection of fetal cells in maternal peripheral blood. Many innovative technologies have been developed to alleviate the burden of scanning large numbers of cells and allow rapid and precise detection of rare events using an automated slide-scanning device and imageanalysis software $[20,21]$. A robust system allowing detection of one male fetal cell or one trisomic 21 cell among 10,000 to 100,000 maternal cells would be extremely useful. Such system would obviate the need or at least significantly reduce the required level of enrichment of fetal cells and facilitate screening large number of slides, making prenatal diagnosis more easily achievable [19]. Evaluation of the efficiency of these automatic slide-scanning devices is mandatory before clinical implementation. Different investigators have already tried to validate automatic scanning devices for the detection of fluorescent signals of rare cellular events [1, 6, 22, 23]. When detection of extremely rare cellular events is required, an accurate evaluation is difficult to obtain. Some groups worked on real clinical samples and compared the results of automatic and manual scanning [20, 22]. However, the accuracy of the manual scanning, which is considered the gold standard, in the detection of rare cellular events had never been validated. Other studies measured the detection efficiency by using prediluted artificial sample mixtures (spiked samples) [1, 19, 20, 22-24]. Dilutions of target cells within a whole cell population are reliable within certain limits of dilution. Nevertheless, in case of fetal cells, an average of 2 to 6 cells $/ \mathrm{mL}$ have been located by manual scanning of 20 to 30 slides with an average of 100,000 cells per slide [9]. In such situations, where the target cells represent an extremely low proportion with an average required dilution of more than $1: 10^{5}$, the predilution strategy seems imprecise and could be considered as an approximation of the real situation. In summary, our protocol can be an accurate tool for the comparison of manual and automatic scanning and the development and validation of the latter for the detection or rare events such as fetal cells in the maternal circulation. 


\section{Conclusion}

Our current investigation indicates that a small amount of circulating male fetal cells dispersed in thousands of female cells can be detected with high specificity and sensitivity using FISH and manual scanning. However, the FISH technique was responsible for missing of $4 \%$ of cells due to nonhybridization or inadequate signaling while $11.5 \%$ were missed as a drawback of the process of manual scanning. Even if the accuracy of manual scanning for signal counting is good, speed and reliability of manual scanning is dependent on technical expertise. This methodology allowed us to determine the efficiency of detection of rare cell events by manual scanning. It establishes a standard for testing new detection strategies of rare event such as fetal cells in the maternal circulation using automatic scanning.

\section{Acknowledgments}

This study was supported by an operating grant from the Canadian Institute for Health Research (CIHR) and Ikonisys through the University-Industry Program to K. Krabchi and R. Drouin. A. Emad is a student scholar of the Public Health Ministry of Egypt. R. Drouin holds the Canada Research Chair in Genetics, Mutagenesis and Cancer.

\section{References}

[1] G. Méhes, A. Luegmayr, C. M. Hattinger et al., "Automatic detection and genetic profiling of disseminated neuroblastoma cells," Medical and Pediatric Oncology, vol. 36, no. 1, pp. 205209, 2001.

[2] J. Al-Maghrabi, L. Vorobyova, A. Toi, W. Chapman, M. Zielenska, and J. A. Squire, "Identification of numerical chromosomal changes detected by interphase fluorescence in situ hybridization in high-grade prostate intraepithelial neoplasia as a predictor of carcinoma," Archives of Pathology and Laboratory Medicine, vol. 126, no. 2, pp. 165-169, 2002.

[3] M. Partridge, R. Brakenhoff, E. Phillips et al., "Detection of rare disseminated tumor cells identifies head and neck cancer patients at risk of treatment failure," Clinical Cancer Research, vol. 9, no. 14, pp. 5287-5294, 2003.

[4] K. Krabchi, M. Gadji, J. C. Forest, and R. Drouin, "Quantification of all fetal nucleated cells in maternal blood in different cases of aneuploidies," Clinical Genetics, vol. 69, no. 2, pp. 145154, 2006.

[5] K. Krabchi, M. Gadji, O. Samassekou, M. C. Grégoire, J. C. Forest, and R. Drouin, "Quantification of fetal nucleated cells in maternal blood of pregnant women with a male trisomy 21 fetus using molecular cytogenetic techniques," Prenatal Diagnosis, vol. 26, no. 1, pp. 28-34, 2006.

[6] S. Hennerbichler, P. M. Kroisel, H. Zierler et al., "Fetal nucleated red blood cells in peripheral blood of pregnant women: detection and determination of location on a slide using laser-scanning cytometry," Prenatal Diagnosis, vol. 23, no. 9, pp. 710-715, 2003.

[7] E. Parano, E. Falcidia, A. Grillo et al., "Noninvasive prenatal diagnosis of chromosomal aneuploidies by isolation and analysis of fetal cells from maternal blood," American Journal of Medical Genetics, vol. 101, no. 3, pp. 262-267, 2001.
[8] A. Sekizawa, Y. Purwosunu, R. Matsuoka et al., "Recent advances in non-invasive prenatal DNA diagnosis through analysis of maternal blood," Journal of Obstetrics and Gynaecology Research, vol. 33, no. 6, pp. 747-764, 2007.

[9] K. Krabchi, F. Gros-Louis, J. Yan et al., "Quantification of all fetal nucleated cells in maternal blood between the 18th and 22nd weeks of pregnancy using molecular cytogenetic techniques," Clinical Genetics, vol. 60, no. 2, pp. 145-150, 2001.

[10] P. L. Kuo, "Frequencies of fetal nucleated red blood cells in maternal blood during different stages of gestation," Fetal Diagnosis and Therapy, vol. 13, no. 6, pp. 375-379, 1998.

[11] H. Mohamed, J. N. Turner, and M. Caggana, "Biochip for separating fetal cells from maternal circulation," Journal of Chromatography A, vol. 1162, no. 2, pp. 187-192, 2007.

[12] E. D’Souza, S. Kulkarni, R. B. Colah, and D. Mohanty, "An improved flow cytometric approach for isolation of fetal cells from maternal blood for non invasive prenatal diagnosis of hemoglobinopathies," Hemoglobin, vol. 31, no. 1, pp. 39-48, 2007.

[13] S. Wada and M. Kitagawa, "Method of separation and concentration of fetal nucleated red blood cells in maternal blood and its application to fetal diagnosis," Congenital anomalies, vol. 44, no. 2, pp. 72-78, 2004.

[14] F. Z. Bischoff, D. A. Marquéz-Do, D. I. Martinez et al., "Intact fetal cell isolation from maternal blood: Improved isolation using a simple whole blood progenitor cell enrichment approach (RosetteSep)," Clinical Genetics, vol. 63, no. 6, pp. 483-489, 2003.

[15] L. P. Shulman, O. P. Phillips, E. Tolley, D. Sammons, and S. S. Wachtel, "Frequency of nucleated red blood cells in maternal blood during the different gestationial ages," Human Genetics, vol. 103, no. 6, pp. 723-726, 1998.

[16] K. Krabchi, M. Gadji, J. Yan, and R. Drouin, "Dual-color PRINS for in situ detection of fetal cells in maternal blood," Methods in Molecular Biology, vol. 334, pp. 141-149, 2006.

[17] S. Mergenthaler, T. Babochkina, V. Kiefer, O. Lapaire, W. Holzgreve, and S. Hahn, "FISH analysis of all fetal nucleated cells in maternal whole blood: improved specificity by the use of two Y-chromosome probes," Journal of Histochemistry and Cytochemistry, vol. 53, no. 3, pp. 319-322, 2005.

[18] R. Drouin, N. Lemieux, and C. L. Richer, "High-resolution Rbanding at the 1250-band level. 1. Technical considerations on cell synchronization and R-banding (RHG and RBG)," Cytobios, vol. 56, no. 225, pp. 107-125, 1988.

[19] J. Yan, E. Guilbault, J. Massé et al., "Optimization of the fluorescence in situ hybridization (FISH) technique for high detection efficiency of very small proportions of target interphase nuclei," Clinical Genetics, vol. 58, no. 4, pp. 309$318,2000$.

[20] K. L. Johnson, H. Stroh, K. Khosrotehrani, and D. W. Bianchi, "Spot counting to locate fetal cells in maternal blood and tissue: a comparison of manual and automated microscopy," Microscopy Research and Technique, vol. 70, no. 7, pp. 585-588, 2007.

[21] M. W. Kilpatrick, T. Tafas, M. I. Evans et al., "Automated detection of rare fetal cells in maternal blood: eliminating the false-positive XY signals in XX pregnancies," American Journal of Obstetrics and Gynecology, vol. 190, no. 6, pp. 1571-1581, 2004.

[22] S. K. Kraeft, A. Ladanyi, K. Galiger et al., "Reliable and sensitive identification of occult tumor cells using the improved rare event imaging system," Clinical Cancer Research, vol. 10, no. 9, pp. 3020-3028, 2004. 
[23] S. Bajaj, J. B. Welsh, R. C. Leif, and J. H. Price, "Ultra-rareevent detection performance of a custom scanning cytometer on a model preparation of fetal nRBCs," Cytometry, vol. 39, no. 4, pp. 285-294, 2000.

[24] T. G. Ntouroupi, S. Q. Ashraf, S. B. McGregor et al., "Detection of circulating tumour cells in peripheral blood with an automated scanning fluorescence microscope," British Journal of Cancer, vol. 99, no. 5, pp. 789-795, 2008. 

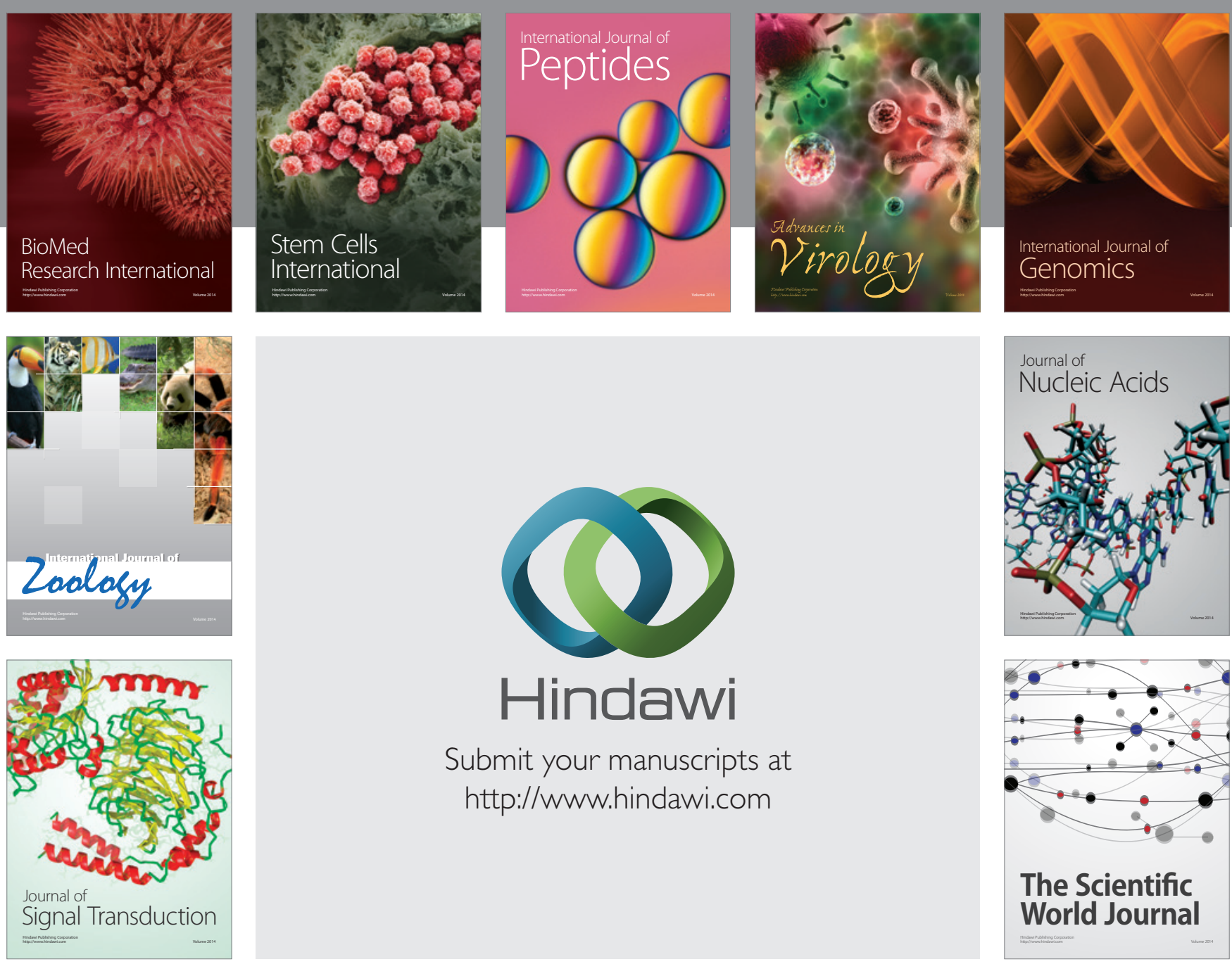

Submit your manuscripts at

http://www.hindawi.com
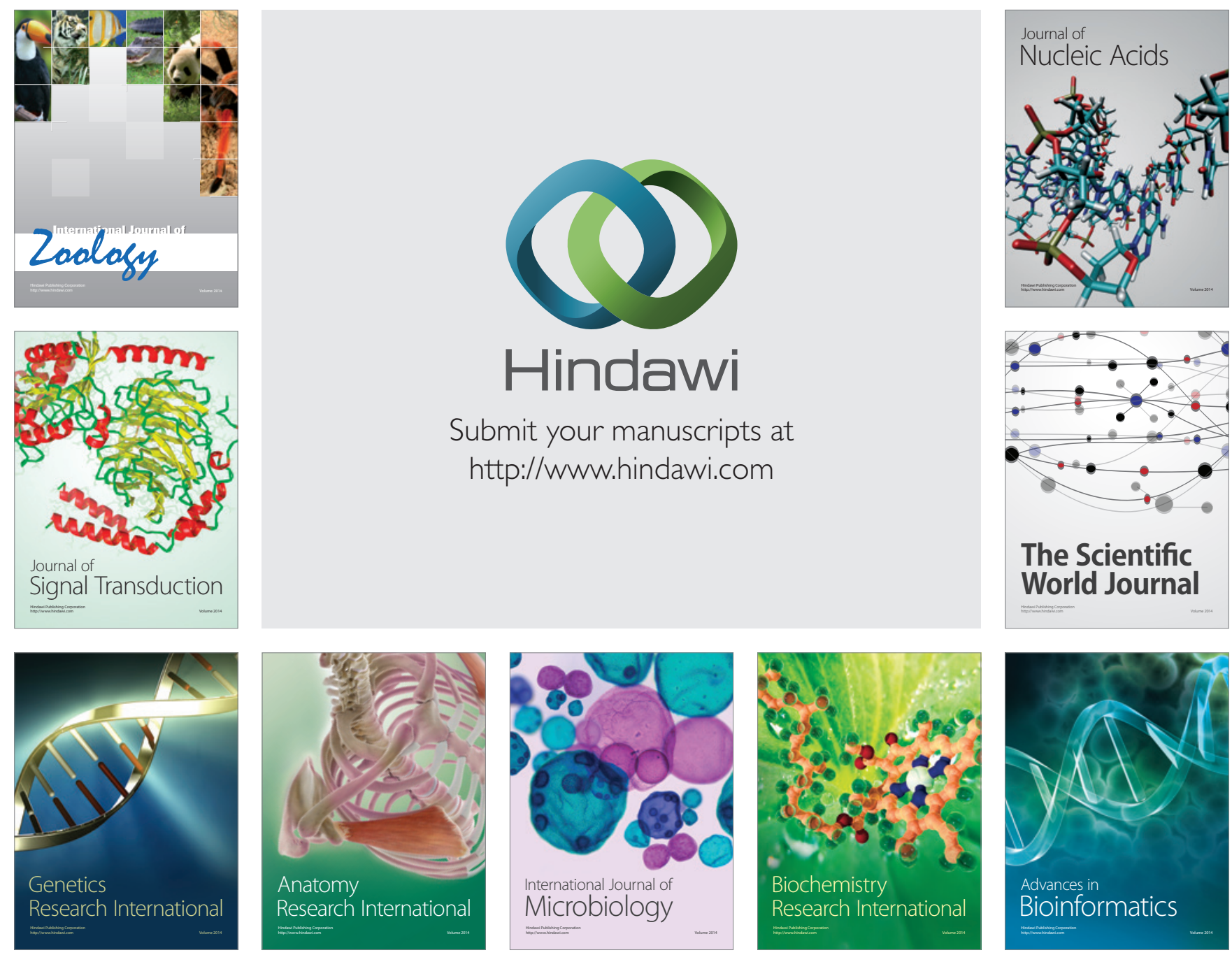

The Scientific World Journal
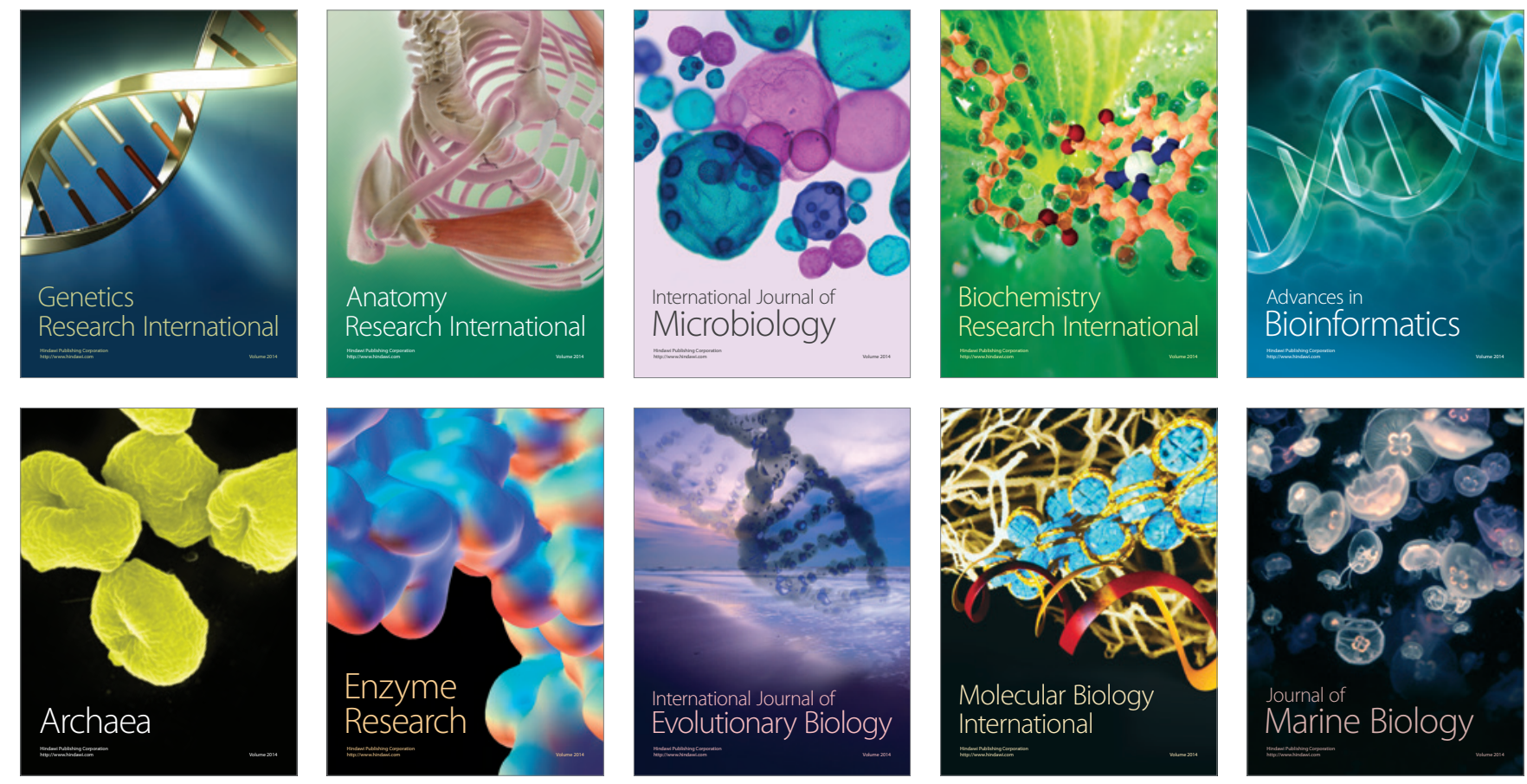